which are bright enough to detect on Earth.

Intriguingly, the effects observed in the black-widow pulsar are similar to distortions seen in pulses from at least one source of fast radio bursts ${ }^{9}$. Plasma lensing might therefore be responsible for boosting the brightness of fast radio bursts, as has previously been hypothesized and modelled ${ }^{10}$. However, the story is not complete: the environment around a source of fast radio bursts is probably quite different from, and possibly even more extreme than, that of the black-widow pulsar. It perhaps has more in common with the environment around the young Crab pulsar or at the centre of our Galaxy ${ }^{11}$.

Main et al. have detected plasma lensing in a pulsar that has been studied for more than 30 years, using a telescope that has been operating since the early 1960s. Why the sudden insight? As computing and data-recording power has grown, so has the ability to use venerable radio telescopes to scrutinize pulsars on shorter timescales and over a wider range of radio frequencies. This suggests that the future is bright for using pulsars to illuminate the invisible Universe.

Jason Hessels is at ASTRON (the Netherlands Institute for Radio Astronomy) and the Anton Pannekoek Institute for Astronomy, University of Amsterdam, 1098XH Amsterdam, the Netherlands. e-mail:j.w.t.hessels@uva.nl

1. Rickett, B. J. Annu. Rev. Astron. Astrophys. 28, 561-605 (1990).

2. Main, R. et al. Nature 557, 522-525 (2018).

3. Fruchter, A. S., Stinebring, D. R. \& Taylor, J. H. Nature 333, 237-239 (1988).

4. Clegg, A. W., Fey, A. L. \& Lazio, T. J. W. Astrophys. J. 496, 253-266 (1998).

5. Hessels, J. W. T. et al. Science 311, 1901-1904 (2006).

6. Romani, R. W., Blandford, R. D. \& Cordes, J. M. Nature 328, 324-326 (1987).

7. Bannister, K. W. et al. Science 351, 354-356 (2016).

8. Backer, D. C., Wong, T. \& Valanju, J. Astrophys. J. 543, 740-753 (2000).

9. Spitler, L. G. et al. Nature $\mathbf{5 3 1}, \mathbf{2 0 2 - 2 0 5}$ (2016).

10.Cordes, J. M. et al. Astrophys. J. 842, 35 (2017).

11. Michilli, D. et al. Nature 553, 182-185 (2018).

\title{
Rethinking WNT signalling
}

\section{The identification of genetic mutations that can hinder the development of human limbs has led to the discovery of an unanticipated mode of regulation for the WNT signalling pathway during limb development. SEE LETTER P.564}

during embryonic development to maintenance and regulation of stem cells in adult tissue. WNTs signal through several distinct intracellular pathways, but these pathways share an initial step: WNT molecules outside the cell bind to and activate receptors of the Frizzled family that span the cell membrane. Frizzled receptors must be present in the membrane for intracellular WNT-pathway activation.

In vertebrates, an auxiliary regulatory process is involved in controlling the accumulation of Frizzled receptors, and hence in determining WNT signalling levels. The system involves three groups of proteins ${ }^{2,3}$ : an LGR (LGR4, 5 or 6), an extracellular R-spondin (RSPO1, 2, 3 or 4), and an E3 ubiquitin ligase enzyme (ZNRF3 or RNF43). In the absence of RSPOs, the ubiquitin ligase tags Frizzled receptors with ubiquitin molecules, which mark the receptors for degradation. This results in low membrane concentrations of Frizzled, so WNT signalling is attenuated ${ }^{4-6}$ (Fig. 1a). Conversely, when RSPOs are present, they bind to LGRs ${ }^{2,3}$, and the resulting complex binds the ubiquitin ligase and prevents it from tagging Frizzled. The receptors accumulate, and WNT signalling can occur ${ }^{4-6}$ (Fig. 1b).

This mechanism has a crucial role in regulating WNT signalling in stem-cell compartments characterized by LGR expression, for instance in the intestine ${ }^{7}$ and hair follicles ${ }^{8}$. Because WNT signalling has been extensively studied in these contexts, the LGR-RSPOligase complex has become part of the standard picture of how WNT activity is regulated.

Szenker-Ravi and colleagues' study began with a genetic analysis of five families affected by either tetra-amelia syndrome, which is characterized by the lack of all four limbs and by lung abnormalities, or by a previously undescribed syndrome involving severe limb malformations. The authors found that these two syndromes are caused by five mutations in the RSPO2 gene that disrupt different protein domains. Using in vitro assays, the researchers demonstrated that the mutations prevent RSPO2 from binding to LGR or RNF43, and so inhibit WNT signalling.

So far, Szenker-Ravi and co-workers' data whose activity controls many processes, from tissue organization and body-axis formation they interact - are known. On page 564, Szenker-Ravi et al. ${ }^{1}$ remind us that this is not necessarily the case.

WNT proteins are signalling molecules
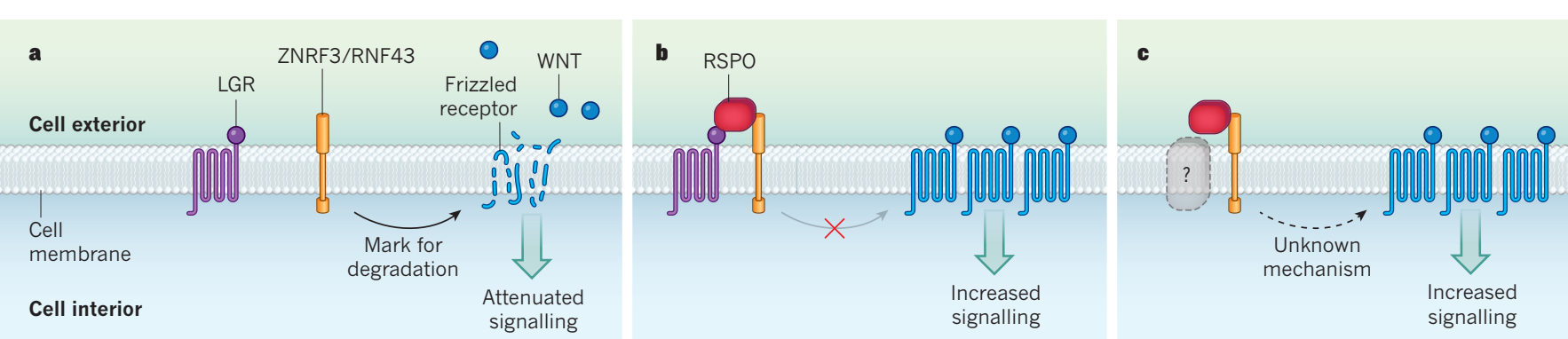

Figure 1 | Updated model of WNT-signalling regulation. WNT signalling, which is triggered when WNT proteins bind to Frizzled receptors that span the cell membrane, is conventionally thought to be regulated by interactions between three groups of proteins: R-spondins (RSPO1 to 4), LGRs (LGR4 to 6) and ubiquitin ligase enzymes (ZNRF3 or RNF43). a, In the absence of RSPOs, LGR and ZNRF3 or RNF43 do not interact, and the ubiquitin ligase catalyses a reaction that marks Frizzled receptors for degradation. WNTs

cannot bind the degraded Frizzled, and WNT signalling is hampered. b, When present, RSPO binds LGRs and ubiquitin ligase, preventing enzyme activity. This allows Frizzled receptors to accumulate and so increases WNT signalling. c, Szenker-Ravi et al. ${ }^{1}$ report that, in the developing limb and lung, RSPO can bind ubiquitin ligase without LGRs. They propose that another, unidentified, protein enables this interaction, which leads to increased WNT signalling through an unknown mechanism. 
fit nicely with the known roles of RSPOs, LGRs and ubiquitin ligases. And, like people carrying $R S P O 2$ mutations, mice lacking Rspo2 have limb abnormalities ${ }^{9}$. The authors expected that the loss of LGR activity would have the same effect. But they got a surprise when they analysed mice lacking the Lgr4, 5 and 6 genes - the triple-mutant embryos did not have limb or lung abnormalities. This suggests that, in some tissues, RSPO2 (and perhaps other RSPOs) can act independently of LGRs, potentiating WNT signalling in the absence of its usual binding partner.

To test this idea directly, the group next investigated whether cells isolated from LGR triple-mutant embryos are capable of RSPOmediated WNT signalling. They found no evidence of WNT signalling when these cells were exposed to Rspo1 or Rspo4, but WNT activity was detected in the presence of Rspo2 or Rspo3. Thus, RSPO2 and RSPO3 seem to be able to induce WNT signalling independently of LGRs. However, these RSPOs still seem to act through their normal ubiquitin ligase targets, because Szenker-Ravi et al. found that modulation of ZNRF3 alters WNT signalling in triple-mutant cells. Consistent with this picture, the authors showed that deletion of rspo2 in the frog Xenopus laevis led to missing limbs, whereas deletion of the $z n r f 3$ and $r n f 43$ genes led to extra limbs.

This study demonstrates that the accepted model of WNT-receptor modulation does not hold in the case of limb and lung development. Szenker-Ravi et al. hypothesize that a separate, unidentified receptor is necessary for this LGR-independent WNT signalling (Fig. 1c). Notably, a study published earlier this year ${ }^{10}$ identified one potential candidate. That work showed that RSPO2 and RSPO3 can bind to ZNRF3 or RNF43 in conjunction with heparin sulfate proteoglycan (HSPG) molecules in lieu of LGRs, to enable WNT signalling in vitro. Future work will be required to test whether HSPGs play this part in the context of lung and limb development. In addition, it remains to be determined whether the HSPG-RSPOZNRF3 complex promotes WNT signalling by preventing ZNRF3 activity, or whether another mechanism is at work. Either way, it will be important to determine the extent of any functional similarities between LGR- and HSPGbased complexes, and to uncover whether there is any pattern to the use of LGR or HSPG as a cofactor in a particular tissue.

Szenker-Ravi and colleagues' work also points to ways to broaden our understanding of processes that require WNT signalling, such as limb development. For example, analysis of the early stages of limb development in frog embryos lacking $z n r f 3$ and $r n f 43$ could reveal why these mutations lead to extra limbs. Do ZNRF3 and RNF43 act as 'master regulators' of limb numbers, as the authors propose? Consistent with this idea, WNT activity has a role in initiating the formation of the limb bud $^{11}$ (which eventually gives rise to the limb).
Alternatively, rather than being master regulators, these proteins might mediate limb numbers indirectly. For example, extra limbs might arise as a secondary consequence of expansion of the pool of limb progenitor cells, or they might arise because of changes in the formation of a signalling centre at the tip of the limb bud that directs limb outgrowth - both WNT-dependent processes ${ }^{12,13}$.

Finally, it will be interesting to evaluate LGRindependent, RSPO-mediated WNT signalling in cancer. Chromosomal abnormalities that lead to activation of RSPO2 or RSPO3 have been shown to drive WNT-dependent colon tumours ${ }^{14}$. Szenker-Ravi and colleagues' demonstration that these two RSPOs can modulate WNT activity independent of LGR adds a twist to these findings, and should prompt scientists to look for cancer-causing mutations in $\mathrm{RSPO} 2$ or RSPO3 in cells outside LGR-expressing cell compartments.

Jessica A. Lehoczky is in the Department of
Orthopedic Surgery, Brigham and Women's Hospital, Harvard Medical School, Boston, Massachusetts 02115, USA. Clifford J. Tabin is in the Department of Genetics, Harvard Medical School. e-mails: jlehoczky@bwh.harvard.edu; tabin@genetics.med.harvard.edu

1. Szenker-Ravi, E. et al. Nature 557, 564-569 (2018).

2. Chen, P.-H., Chen, X., Lin, Z., Fang, D. \& He, X. Genes Dev. 27, 1345-1350 (2013).

3. Gong, X. et al. PLOS ONE 7, e37137 (2012).

4. Hao, H.-X. et al. Nature 485, 195-200 (2012)

5. Carmon, K. S., Gong, X., Lin, Q., Thomas, A. \& Liu, Q. Proc. Natl Acad. Sci. USA 108, 11452-11457 (2011).

6. de Lau, W. et al. Nature 476, 293-297 (2011).

7. Sato, T. et al. Nature 459, 262-265 (2009).

8. Jaks, V. et al. Nature Genet. 40, 1291-1299 (2008).

9. Bell, S. M. et al. Development 135, 1049-1058 (2008).

10.Lebensohn, A. M. \& Rohatgi, R. eLife 7, e33126 (2018).

11.Kawakami, Y. et al. Cell 104, 891-900 (2001).

12.ten Berge, D., Brugmann, S. A., Helms, J. A. \&

Nusse, R. Development 135, 3247-3257 (2008).

13. Kengaku, M. et al. Science 280, 1274-1277 (1998).

14. Han, T. et al. Nature Commun. 8, 15945 (2017).

This article was published online on 16 May 2018.

\section{ANTHROPOLOGY}

\section{Sizing up human brain evolution}

\section{An innovative computational analysis of factors that might have influenced human brain evolution suggests that ecological, rather than social, factors had a key role in the evolution of large, rapidly developing brains. SEE LETTER P.554.}

\section{RICHARD MCELREATH}

$\mathrm{M}$ ost organisms are brainless but thriving. Brains are expensive to produce and maintain, and in the human lineage they have grown so large as to incur a substantial metabolic burden as the brain develops ${ }^{1}$. A human brain stops growing by the age of ten, long before the body reaches physical maturity, and this costly and fast process of brain growth has been proposed to cause a delay in body growth ${ }^{1}$. Brain growth is not given priority in this way in other apes, and the human pattern is puzzling because it keeps our bodies smaller, more vulnerable and less productive for longer. The answer to this riddle must lie in how the human brain helped our ancestors to survive and reproduce. On page 554, González-Forero and Gardner ${ }^{2}$ investigate the role of different factors as possible drivers of our unusually large brains, and determine how well these factors might account for the pattern of changes in brain and body size that occur as humans develop.

Proposals for how large brains evolved in humans include ecological, social and cultural hypotheses. The ecological-intelligence hypothesis suggests that environmental challenges, such as finding food, are paramount in driving brain-size evolution ${ }^{3}$. The social-intelligence hypothesis suggests instead that the competitive and cooperative challenges of living with other members of the same species are the key factor ${ }^{4}$. The cultural-intelligence hypothesis combines these two ideas, suggesting that the social learning of ecologically relevant skills explains the extreme brain investment of our lineage ${ }^{5}$.

Until now, testing these hypotheses has relied mainly on comparative studies that correlate data on brain characteristics such as size (as an approximation of intelligence) with features such as cognition, ecology and group living. These regression approaches, which seek to identify variables that are associated with brain size, have been valuable for refining theories and the data measurements needed.

However, such regression studies can generate conflicting and confusing results. Changes to brain and body growth can have a reciprocal effect on each other for various reasons, such as metabolic constraints and energy-production needs, so such interactions between the brain and the body are complex and nonlinear. This makes the results of regression studies hard to interpret, because they cannot be connected directly to a relevant 